\title{
A natureza categorial da preposição
}

\author{
Márcia Barreto Berg \\ Fundação de Ensino Superior de São João del-Rei
}

\section{Abstract}

The aim of this paper is to identify the categorial nature of preposition, from the perpective of Chomsky's Generative Grammar (1981/1995).

The preposition has been described as a lexical, uniform category, defined by the features [ N, V]. This classification is not consensual, however. We therefore made a survey of the proprieties that distinguish the lexical from the functional, applied the properties to the data of Brazilian Portuguese and came to conclusion that prepositions belong to the functional category. 
T dentificar a natureza categorial das preposições é nosso objeto de estudo. O referencial teórico aqui adotado constitui-se de L hipóteses desenvolvidas no Programa de Pesquisa da Gramática Gerativa (Chomsky, 1981/1995).

Assim como os nomes, verbos e adjetivos, a preposição tem sido descrita como uma categoria uniforme do tipo lexical, cujos traços são [+N, +V] (Chomsky, 1970/74). Esta classificação, entretanto, não é consensual. Há estudiosos que colocam em dúvida se:

1) em todos os seus usos, as chamadas preposições são núcleo de PP;

2) se os limites entre elas e outras categorias é claro e;

3) se as preposições seriam uma categoria do tipo lexical.

Explicaremos, resumidamente, em separado, os itens acima. Antes, porém, gostaríamos de justificar a nossa motivação em elucidar a natureza categorial das preposições.

Com as grandes mudanças de transição na sintaxe gerativa para a abordagem princípios e parâmetros, o papel e a natureza das categorias funcionais foram repensados. A visão que surgiu começa a inverter a valorização tradicional, atribuindo agora o papel central na estrutura frasal aos elementos funcionais. A noção de categoria "funcional" em oposição à "lexical" se reveste, portanto, de cada vez mais importância dentro do quadro da gramática gerativa. O pressuposto de que o núcleo de toda projeção é funcional (Fukui, 1986; Fukui \& Speas, 1986) atribui a essas categorias um papel fundamental na concepção da gramática. Além disso, as categorias funcionais são vistas como definidoras das gramáticas particulares: "a variação paramétrica se reduz, em grande parte, à variação nas propriedades lexicalmente especificadas das categorias funcionais" (Ouhalla, 1991: 3). Sugere-se até que há sistemas cerebrais diferentes 
para lidar com categorias funcionais e categorias lexicais (Tsimpli \& Ouhalla, 1990), apud Ouhalla, 1991, p. 10, o que recebe o apoio de evidências oriundas de síndromes patológicas (Grodzinsky, 1990). Também na psicologia a distinção entre funcional e lexical é importante, pois, na aquisição da linguagem, as crianças adquirem elementos funcionais mais tarde que os lexicais. Já em certas afasias, a habilidades de processar elementos funcionais é perdida enquanto o uso e entendimento dos elementos temáticos (= lexicais) sobrevive (Abney, 1987: 64).

Agora vamos à explicação dos itens (1 a 3) relacionados acima.

\section{CENÁRIO ATUAL: PREPOSIÇÕES E PREPOSIÇÕES?}

\subsection{O tipo de categoria a que a preposição pertence}

Na literatura há controvérsias de que a preposição seja lexical ou funcional. Abney (1987: 63) apresenta dúvidas quanto à categoria da preposição ao afirmar que "P seems to straddle the line between functional and thematic elements; one might wish to treat it as unspecified for [+ F]". Nesse texto, onde se lê "thematic" entendese por "lexical" e onde se lê "grammatical" entende-se por "funcional.

Já Emonds (1985: 14) sugere que a preposição pertença à classe funcional "P (which usually corresponds to the tradicional term 'preposition') is a head which is a grammatical rather than a lexical cathegory".

Há também autores que implicitamente colocam em dúvida o estatuto da preposição, ao classificá-la como vazia de significado. Essa classificação recebe por alguns autores o nome de "prepositions dummy": Williams (1989), Marantz (1984), Giorge (1991), Borer (1985) dentre outros. Dummy implica na propriedade de não atribuir papel temático.

Chomsky (1986) trata esse tipo de preposição, que ele identifica como "of", como realizadora de Caso e não atribuidora de Caso, o que implica rejeitar o seu estatuto como categoria lexical, pois para ele, todas as categorias lexicais atribuem Caso. 


\subsection{Os traços que definem a categoria preposição}

Chomsky considera a visão de Port Royal para definir as categorias sintáticas principais em termos de duas noções semânticas:

$$
\begin{aligned}
& \text { Substantiva }=[+\mathrm{N}]=\text { argumento } \\
& \text { Predicativa }=[+\mathrm{V}]=\text { predicado }
\end{aligned}
$$

Segundo Chomsky (1981) o traço [V] corresponde à propriedade de atribuir Caso e papel- $\theta$ e o traço [N] corresponde à propriedade de não atribuir Caso e papel- $\boldsymbol{\theta}$. Se a preposição é definida pelos traços [-N, -V], à luz de Chomsky (1981) podemos concluir que:

$$
\begin{aligned}
& {[-\mathrm{V}]=[- \text { Caso },-\theta]=[- \text { predicado }]} \\
& {[-\mathrm{N}]=[+ \text { Caso },+\theta]=[\text {-argumento }]}
\end{aligned}
$$

Desse modo a descrição em termos de [-N] e [-V] indica que a preposição não é nem argumento, nem predicado, gerando dúvidas quanto ao seu estatuto lexical.

\subsection{A uniformidade da preposição}

Se a preposição é uma categoria uniforme, seria de se esperar que todas tivessem um comportamento semelhante, isto é, todas preposições deveriam preencher o lugar de núcleo do PP, entretanto ela pode ser considerada núcleo de IP, quando "to" é marcada pelo traço [- tempo] (Haegman, 1991). Freidin (1992) insere a preposição "of" , das estruturas nominais, debaixo de NP. Chomsky (1981), considera "for" [-wh] como $\mathrm{C}^{\mathrm{O}}$, assim como Williams \& Dubinsky (1995) que admitem que as preposições temporais ocupam a posição de $\mathrm{C}^{\mathrm{O}}$ quando ocorrem antes de um complemento oracional. Lobato (1989) apresenta dúvidas de qual elemento é o núcleo na expressão "atrás de", visto que Lemle (1984) considera o advérbio "atrás" como uma preposição. Uriagereka (1995) coloca a preposição como núcleo de AGR. 
Assim, com a diversidade das propostas apontadas acima, não ficam claras as fronteiras entre: (a) P e advérbio; (b) P e Complementizador; (c) P e Nome; (d) P e tempo.

\section{AS CATEGORIAS LEXICAIS E FUNCIONAIS}

Para tentar resolver os problemas acima e definir o estatuto categorial da preposição, fizemos um levantamento das propriedades que distinguem o que seja uma categoria lexical e funcional. Esse levantamento foi baseado nos trabalhos de Fukui \& Speas (1986), Abney (1987), Ouhalla (1991) e Chomsky (1995). Depois de analisar e comparar as propriedades das referidas categorias, generalizamos ainda mais essas propriedades estabelecendo um quadro definidor:

\section{(quadro 1)}

\begin{tabular}{|l|l|}
\hline \multicolumn{1}{|c|}{ Categorias funcionais } & Categorias lexicais \\
\hline 1) Representam uma classe fechada & 1) Representam uma classe aberta \\
\hline $\begin{array}{c}\text { 2) Apresentam uma contribuição } \\
\text { semântica de segunda ordem }\end{array}$ & $\begin{array}{c}\text { 2) Apresentam uma contribuição } \\
\text { semântica plena. }\end{array}$ \\
\hline 3) Não atribuem papel temático & 3) Atribuem papel temático \\
\hline 4) Atribuem/Checam caso & 4) Não atribuem/Checam caso \\
\hline
\end{tabular}

Apesar das limitações do diagnóstico feito a partir dos textos resenhados e assumindo que a distinção lexical e funcional permite identificar metacategorias, passamos a investigar em que metacategorias as preposições podiam ser incluídas.

Essas propriedades foram aplicadas aos dados do Português do Brasil. Para efeito de argumentação assumi, como hipótese nula de trabalho, que as preposições são categorias lexicais. Nessas bases, as preposições do Português do Brasil deveriam: 
a) não formar uma classe fechada e sim formar uma classe aberta;

b) possuir uma contribuição semântica plena;

c) atribuir papel temático;

d) não atribuir/checar Caso.

Assumiremos, antecipadamente que as categorias lexicais não atribuem Caso, visto que, de acordo com Chomsky (1995), a noção de atribuição é substituída pela noção de checagem. Somente as categorias funcionais checam caso. Assim N, A e V não atribuem Caso (o Caso objetivo é checado por $\mathbf{v}$ e não por $\mathbf{V}$ ). Nessas bases somente $\mathrm{P}$ atribuiria Caso, porém mostraremos no final deste trabalho que P não é uma categoria lexical e sim funcional.

Em direção à nossa hipótese nula de trabalho: "Preposição é uma categoria Lexical", mostraremos em (3), abaixo, que as propriedades que dão suporte a essa hipótese não se confirmam.

\section{CHECAGEM DAS PROPRIEDADES}

\subsection{A preposição: classe fechada?}

As informações contidas nesta seção foram buscadas em estudos de abordagem tradicional, por serem os únicos que discutem explicitamente a questão relativa a que itens formam o conjunto das preposições. Trabalhamos apenas com as preposições consideradas "essenciais". Nesse trabalho não abordamos as locuções prepositivas.

O quadro a seguir é o resumo de uma listagem de preposições essenciais apresentada por diversos gramáticos: Ali (1964), Almeida (1988), André (1990), Bechara (1992), Cegalla (1994), Cunha (1986), Lima (1984), Luft (1989), Sacconni (1979). Separamos os autores em quatro grupos, de acordo com o número de preposições que cada um listava. 


\section{0 grupo: Preposições listadas por todos os autores \\ Número total das preposições listadas $=19$}

A, ante, após, até, com, contra, de, dês, desde, em, entre, para, per, perante, por, sem, sob, sobre, trás.

\begin{tabular}{|c|c|c|}
\hline \multicolumn{2}{|c|}{ Preposições não-comuns aos diversos autores } \\
\hline $\begin{array}{l}\mathbf{2}^{\mathbf{o}} \text { grupo } \\
\text { dês }\end{array}$ & $\begin{array}{c}\mathbf{3}^{\mathbf{o}} \text { grupo } \\
\text { dês, per }\end{array}$ & $\begin{array}{c}\mathbf{4}^{\mathbf{o}} \text { grupo } \\
\text { dês, per, trás }\end{array}$ \\
\hline \multicolumn{2}{|c|}{ Número das preposições comuns a todos os autores $=\mathbf{1 6}$} \\
$\begin{array}{l}\text { A, ante, após, até, com, contra, de, desde, em, entre, para, perante, por, sem, } \\
\text { sob, sobre }\end{array}$
\end{tabular}

A comparação das listagens extensionais acima permite um quase total consenso quanto aos itens da classe das preposições. Dezenove (19) é o número máximo de elementos enumerados como componentes dessa classe. O mínimo é dezesseis (16) elementos.

As preposições "dês", "per" e "trás", que são preposições nãocomuns a todos autores, são por eles justificadas - preposições que estão em desuso atualmente. Este fato evidencia, por parte dos autores, o reconhecimento de estarem incluindo algumas preposições a mais numa lista fechada. Assim podemos concluir que os elementos pertencentes à classe das preposições do português contemporâneo são de número limitado, formando uma classe fechada e estável. Diferente das outras categorias, não se observam neologismos na categoria das preposições (Alves, 1990). Portanto, em relação à natureza categorial, podemos afirmar que as preposições comportamse como categorias funcionais de acordo com o quadro (1).

\subsection{O valor semântico da preposição}

O segundo item da nossa hipótese de trabalho, o valor semântico da preposição, pode-se dizer que, de modo geral, aceitase que algumas preposições têm conteúdo semântico e outras não 
têm (Giorgi, 1991; Borer, 1981; Chomsky, 1986). Há também autores que assumem essa distinção, embora reconheçam que algumas preposições podem ser vazias em um contexto e não-vazias em outro (Williams, 1989; Williams e Grimshaw, 1993; Lobato, 1989).

Analisando as propostas desses autores, constatamos que os critérios para tal distinção não são muito claros, pois uma mesma preposição pode ser vazia ou semântica, como podemos verificar, abaixo, nos exemplos (1) e (2) de Williams e Grimshaw (1993) e nos exemplos (3) e (4) de Lobato:

(1) The letter to John by himself (to = gramatical)

(2) The letter was to John (to = semântica)

(3) João só viaja com seus amigos (com = Preposição verdadeira)

(4) João só concorda com seus amigos (com = Preposição não-verdadeira)

(Entende-se que "Preposição verdadeira" = preposição semântica e "P. não-verdadeira" = funcional).

Essa ambigüidade nos leva a pensar que a distinção entre $P$ semântica versus $P$ gramatical está relacionada ao lugar na estrutura frasal onde ela foi inserida e não no seu conteúdo intrínseco.

Quanto às preposições do Português do Brasil, dividimo-las em duas subclasses: a) As preposições com mais de um significado e b) As preposições com apenas um significado.

Quanto à letra (a), segundo Cunha (1986), "as preposições embora apresentem grande variedade de usos, bastante diferenciados no discurso, é possível estabelecer para cada uma delas uma significação fundamental (grifo meu), marcada pela expressão de movimento ou de situação resultante e aplicável aos campos espacial, temporal e nocional".

Aplicados testes sintáticos para as preposições $\mathbf{a}, \mathbf{c o m}, \mathbf{d e}, \mathbf{e m}$, para, por, sobre, constatamos que essa significação múltipla é aparente. Exemplificaremos abaixo apenas com a preposição a, por acreditarmos que as outras preposições se inserem no rol dos mesmos critérios com que foi analisada a preposição a. 


\section{(5) Voltei a Ouro Preto \\ (6) Deu ao Pedro \\ (7) Doação ao Pedro}

Celso Cunha atribui à preposição a em (5, 6 e 7) os valores de movimento (= direção a um limite) e situação. Entretanto, parecenos que em (5) a preposição não é responsável pelo conteúdo descritivo de "movimento" e sim o verbo "voltar", que por si denota "movimento", pois se trocarmos o verbo, o sentido de movimento desaparece: Oprefeito pagou a Ouro Preto. Em (6) e (7) parece ser o verbo dar (em forma nominal ou verbal) que traz intrínseco o conteúdo descritivo de benefactivo. Uma evidência de que o sentido está no verbo, é trocarmos somente o verbo, mudando-se assim o sentido do NP: obedeceu ao Pedro/obediência a Pedro.

Outro teste sintático aplicado foi o fato de que as preposições da letra (a) não podem ocorrer no final da sentença:

(8) *Maria falou com.

(9) *Voltei a.

(10) *Pedro pensou em.

Assim, concluímos que a preposição atua com uma significação semântica de segunda ordem, ajudando na interpretação de seu complemento, não sendo, portanto, ela, a preposição sozinha, a responsável pela significação do complemento.

Quanto à letra (b), preposições com apenas um significado, foram aplicados testes sintáticos com as preposições ante, após, até, contra, desde, entre, perante, sem, sob e constatamos que elas têm um comportamento atípico, aproximando-se da classe dos advérbios. Exemplificaremos abaixo apenas com as preposições sem e contra, por acreditarmos que as outras supracitadas em (b) se encontram no mesmo rol dos critérios explicitados em (11 e 12).

(11) Maria lutou a favor d(os comunistas) e Pedro lutou contra.

(12) Maria caminhou com (o lenço) e Pedro caminhou sem. 
As relações que as preposições contra e sem estabelecem são sempre as mesmas, independente do contexto onde aparecem: contra $=$ noção de oposição $/$ sem $=$ subtração, ausência . Outro fato que as diferencia do primeiro grupo é o fato de elas poderem ocorrer no final da sentença.

Adotamos, então, como verdadeiras, apenas as preposições a, com, de, em, para, por, sobre e concluímos que as preposições não são completamente destituídas de significado e nem possuem um conteúdo semântico pleno, mas, sim, apresentam uma contribuição semântica de segunda ordem. Uma evidência disso é o fato de elas não poderem ser apagadas in situ como em "*eu gosto $\varnothing$ bolo" ou “*eu vim $\varnothing$ Paris". Outra evidência é o fato de elas mudarem de sentido quando trocadas no interior da sentença. Do mesmo modo que o tempo indica "passado" e "não-passado", as preposições indicam relações e não, ações ou eventos ou entidades.

Portanto, em relação à nossa hipótese nula, as preposições em (a) comportam-se como categorias funcionais.

\subsection{A atribuição de papel temático}

Quanto ao terceiro item da nossa hipótese nula de trabalho, a atribuição de papel temático, constatamos que a preposição não tem essa propriedade, visto que ela não contém um conteúdo semântico pleno. Entretanto, há na literatura várias propostas:

\section{(1) A preposição atribui papel temático ao NP}

Chomsky (1981, 1986), Pollock (1988) e Borer são alguns exempos de autores que admitem essa proposta. Entretanto, a ausência de contraste entre as sentenças de doce eu gosto / doce eu gosto, é um obstáculo para essa proposta, pois a supressão da preposição deveria fazer com que a estrutura ficasse malformada, já que a transmissão do papel temático não seria possível. 


\section{(2) A preposição transmite papel temático ao NP}

Essa proposta se baseia na afirmação de que o verbo e/ou o nome atribui papel temático ao PP e este o transmite ao NP objeto. Ouhalla, 1994 e Marantz, 1984 sustentam essa proposta. Analisando a proposta desses autores entendemos que o elemento que transmite papel temático, de fato não atribui. Se assim é, podemos afirmar que indiretamente esses autores afirmam que a preposição não atribui papel temático.

\section{(3) A preposição não atribui papel temático ao NP}

A noção de que o verbo/nome é o responsável pela atribuição temática vem acompanhada da discussão de que a preposição é "dummy", i.e., vazia de significado. Todos os autores que admitem a existência de preposições "dummy" admitem indiretamente que elas não atribuem papel temático. Concordamos com esses autores no que diz respeito à tal atribuição. Baseados em Larson (1985), admitimos que o elemento responsável pelo papel temático é um traço (F) que o NP, complemento de P, possui. Esse traço é oriundo das propriedades de seus próprios núcleos.

A fim de tornar mais claro o traço (F), nos remetemos a Larson (op. cit., p. 595) que descreve o NP em (13), abaixo, como "bareNP adverbs".

\section{(13) I saw John $\left[_{\mathrm{NP}}\right.$ that day]}

Larson se baseia na análise de Bresnan e Grimshaw (1978) para justificar atribuição de Caso e papel- $\theta$ "bare-NP adverbs". A classe de NP advérbios é marcada pelo traço F (= tempo, lugar, modo, etc), de acordo com seus significados: "place", "here", "there", etc. Larson adota a idéia de Bresnan e Grimshaw e assume que os NPs possuem um traço $(+\mathrm{F})$ que é oriundo das propriedades de seus próprios núcleos, isto é, herdado por qualquer $\mathrm{NP}$ tendo um $\mathrm{N}$ como seu núcleo. O NP recebe papel- $\theta$ de acordo com sua carga semântica intrínseca (= seu conteúdo semântico inerente).

A meu ver, podemos traduzir a análise de Larson em termos do Programa Minimalista: 
(i) o traço [+F] é um traço semântico intrínseco e

(ii) a noção de "receber papel temático de $[+\mathrm{F}]$ " é equivalente a receber papel temático de $\mathrm{P}$.

Pode-se afirmar então que "receber papel temático de P ou [+F] equivale a dizer que $\mathrm{P}$ ou [F] tornam o NP acessível à computação", visto que se $\mathrm{P}$ ou $[\mathrm{F}]$ não realizam sua função, a estrutura fica malformada, como em (14) abaixo:

\section{(14) Eu vim de Paris}

*Eu vim Ø Paris

O papel da preposição seria então o de tornar o traço [+F] visível quando necessário. É importante ressaltar que a preposição não acrescenta traços ao NP, ela apenas realça, torna proeminente um traço semântico do NP.

Para explicitar o que significa acessível à computação, baseamo-nos em Chomsky (1995): a língua consiste de dois componentes: um léxico e um sistema computacional. O léxico especifica os itens que entram no sistema computacional com suas propriedades idiossincráticas. O sistema computacional usa esses elementos para gerar derivações e Des (descrições estruturais). No sistema computacional há dois níveis de interface: a) perceptuais/ articulatórios $(=\mathrm{FF})$ e b) conceptuais/intensionais (=FL). Uma derivação converge ou é acessível à derivação, se ela converge na FF e FL.

Com relação às preposições, se [+F] for visível (i.e. acessível), o NP (complemento da preposição) é interpretado. Se o traço [+F] não for visível, o NP não é interpretado, como em 14 acima. O papel da preposição seria então o de tornar o traço $[+\mathrm{F}]$ visível, quando necessário.

É importante ressaltar que a preposição não acrescenta traços ao NP, ela apenas realça, torna proeminente um traço semântico do NP.

Outra evidência de que P não atribui papel temático, em contraposição aos verbos, é o fato de ela não selecionar tematica- 
mente seu complemento, podendo coocorrer com qualquer NP: de água/ com água/ sobre água/ em a água/para água/ à água, em contraposição a *dormir água/ *costurar água, beber água, etc..

Com relação aos verbos, há restrições, isto é, nem todo NP pode coocorrer com qualquer verbo, ao passo que, com a preposição, isso não ocorre, ou seja, ela pode coocorrer com qualquer NP.

\subsection{A atribuição/checagem de caso}

O quarto e último item da nossa hipótese nula de trabalho, o Caso, constatamos que as preposições checam o Caso no Spec de PP.

Na literatura mais recente, a noção de atribuição é mudada para Checagem, entretanto, quanto ao Caso objetivo, permanece a noção de que a preposição é que atribui esse Caso ao seu complemento. Os Casos nominativo, acusativo, genitivo e objetivo têm naturezas distintas em relação à Teoria Temática, por isso se distribuem em duas subclasses: casos estruturais e Casos inerentes. A diferença fundamental entre ambos é a seguinte:

"... inherent Case is assigned by $\alpha$ to NP if and only $\alpha \theta$-marks NP, while structural Case is assigned independently of $\theta$-marking" (Chomsky 1986, p.193)

De acordo com essa formulação entende-se que um elemento só pode ser atribuidor de Caso inerente se ele também atribui um papel temático ao NP. Os Casos considerados inerentes são genitivo e oblíquo.

\section{O caso genitivo}

Desde Vergnaug (1980) concebe-se a preposição como uma categoria lexical atribuidora de Caso. Esse estatuto é, de certo modo, fragilizado quando Chomsky (1986, p. 193) argumenta a favor da distinção entre atribuidor/realizador de Caso, nos ambientes onde há Caso genitivo: o adjetivo e o nome são de fato atribuidores de 
Caso genitivo e "of" realiza esse Caso (Chomsky 1995, p.114). Assim a preposição "of" não atribui Caso inerente. O programa Minimalista não deixa clara a questão das preposições. Chomsky adota a teoria da checagem, mas parece que, no que diz respeito às preposições, permanece a noção de atribuição/realização. Fica então um problema não resolvido: se todo NP entra na numeração com um traço de Caso, onde o NP, complemento de P, é checado?

\section{O caso oblíquo}

O Caso oblíquo é concebido como um Caso inerente atribuído por preposição. Por ser inerente, é associado à atribuição de papel temático. Se a análise desenvolvida nas seção "Papel Temático" estiver correta, não haverá lugar para Caso inerente, visto que P não atribui papel temático. Com isso propomos que as preposições tenham o mesmo comportamento de Caso nas estruturas $[\mathrm{V}+\mathrm{P}+\mathrm{N}]$ e $[\mathrm{N}+\mathrm{P}+\mathrm{N}]$ independentemente se é Caso Oblíquo ou genitivo.

Segundo o Programa Minimalista, a configuração para checar Caso é Spec-núcleo. Sabemos que o NP complemento da preposição é checado, porque ele é visível na FL, isto é acessível à derivação. A questão que se suscita é como o traço de $\mathrm{N}$, complemento de $\mathrm{P}$, "bolo" e "casa" como, por exemplo, em (15) e (16) abaixo, poderiam ser checados, já que, para Chomsky, todo NP entra na numeração com um traço de Caso.

(15) Eu gosto de bolo

(15) A construção da casa

Apresentamos então algumas propostas: uma das hipóteses seria nos basearmos em Uriagereka (1995), que propõe uma estrutura de AGRp, cuja preposição ocupa o lugar de núcleo desta estrutura. Assim o traço do NP poderia ser checado no Spec de AGRp. Entretanto é inadequado falar de traços- $\phi$ para as preposições do Português do Brasil.

Uma outra hipótese seria admitir que P tem o mesmo comportamento de D. Assim como D é checado no Spec de DP, P também 
seria checado no Spec de PP. O problema com essa hipótese, é que, nas estruturas possessivas de DP, do tipo "John's picture", o Caso é checado para a esquerda e esse tipo de estrutura não existe no $\mathrm{PB}$ (*John's retrato, e sim retrato de John). Em PB as preposições não licenciam esse tipo de spec.

Seria então mais razoável admitir que o traço do NP complemento de $\mathrm{P}$ é checado no spec do próprio PP. A posição de spec do $\mathrm{PP}$ conteria um traço de Caso onde ocorreria a checagem do traço de Caso do NP.

\section{CONCLUSÃO}

Na introdução deste trabalho, levantamos três questões: (1) a qual o tipo de categoria que a preposição pertence (lexical ou funcional?); (2) quais os traços que a definiriam (= os limites entre a preposição e outras categorias) e (3) a sua uniformidade (= todas preposições são núcleo de PP?). Abaixo responderemos a essas questões com base nas discussões dos itens de (1) a (3) deste trabalho.

4.1 - A natureza categorial da preposição é funcional já que elas têm as seguintes propriedades: a) pertencem à uma classe fechada; b)possuem um conteúdo significativo de segunda ordem; c) não atribuem papel temático e d) atribuem/checam Caso.

4.2 - Os traços que definem a preposição $[-\mathrm{N},-\mathrm{V}]$ não a definem nem como argumento nem como predicado. Assim reinterpretamos Chomsky (1981/1986) e redefinimos [-N, -V] como traços que definem uma categoria funcional. Isto é, [-N] significa não pertencer à categoria dos nomes e [-V] significa não pertencer à categoria dos verbos, logo, a categoria que possui esses traços só pode ser uma categoria funcional.

4.3 - A uniformidade da classe preposição: fizemos um corte subdividindo as preposições do Português do Brasil em dois 
grupos. No primeiro grupo temos os elementos ante, após, contra, perante, sem, sob, desde, entre, que, de acordo com nossas discussões, não se comportam como preposições; e, no segundo grupo, temos os elementos a, com, de, em, para, por, sobre, que consideramos como preposições propriamente ditas. São essas as preposições que ocupam lugares diferentes na estrutura frasal, ocorrendo como núcleos de CP, TP, AGR, DP, SN e PP. Esta é uma característica muito singular que as distingue dos outros elementos.

Vemos algumas vantagens em admitir a preposição como categoria funcional:

(a) Elucidar a dúvida latente em muitos autores, por exemplo, Abney, 1987, Ouhalla, 1991 e o próprio Chomsky, 1981.

(b) A análise uniforme no que diz respeito ao Caso: todas as preposições checariam o Caso estrutural.

Entretanto deixamos em aberto algumas questões para pesquisas futuras:

(a) Qual seria a análise da preposição nas estruturas [oração] $\mathrm{P}$ [oração], com em (16) abaixo?

(16) [Maria gosta] de [cantar no banheiro]

O elemento "de" é um $\mathrm{P}^{\mathrm{O}}$ ou um $\mathrm{C}^{\mathrm{O}}$ ?

(b) Como explicar o contraste entre (1) e (2) abaixo, isto é, por que a preposição só pode ser apagada quando subcategorizada pelo verbo?

(1) De bolo eu gosto / $\phi$ bolo eu gosto

(2) De Paris eu vim / * $\phi$ Paris eu vim

O apagamento da preposição seria, de fato, uma questão da recuperabilidade de seu conteúdo?

(c) Qual é o limite entre a classe de preposições e as outras classes, como, por exemplo os advérbios? 


\section{BIBLIOGRAFIA}

ABNEY, Steven Paul. The English noun phrase in its sentential aspect. Tese de doutorado, MIT, Cambridge, Mass, Junho de 1987.

. Functional elements and licensing. Conferência apresentada em GLOW, Gerona, Espanha, 1986.

ALVES, Ieda Maria. Neologismos. Criação lexical. São Paulo: Ática, 1990.

BORER, H. The syntax of pronominal clitics. Orlando: Academic Press, 1986.

CHOMSKY, Noam. Remarks on nominalization. In: R. Jacobs \& P, Rosenbaum, Waltham, Mass, Ginn, 1970. p. 184-221.

. Lectures on Government and Binding. Dordrecht, Holland, Cinnaminson: Foris Publications, 1981.

. Knowledge of language: its nature, origin and use. New York: Praeger Publishers, 1986. Minimalist Program. Cambrige, Mass, London: MIT Press, 1985.

EMONDS, Joseph E. A unified theory of syntactic categories. Dordrecht: Foris, 1985.

FREIDIN, Robert. Foundations of Generative Syntax. Cambridge, Mass, London: MIT Press, 1992.

FUKUI, N e SPEAS, M. Specifiers and projection. In: Papers in Theoretical Linguistics. Vol. 8, p. 128-172, 1986.

FUKUI, Naoki. A theory of category projection and its applications. Tese de doutorado, MIT, Cambrigde, Mass, junho de 1986.

GIORGI, Alessandra. The syntax noun phrases: configuration, parameters and empty categories. New York: Cambridge, 1991.

GRIMSHAW, Jane e WILLIAMS, Edwin. Nominalization and predicative prepositional phrases. In: Semantic and the lexicon. Dordrecht, j. Pustejovsky, 1993. Cap. 7, p. $97-105$

GRODZINSKY, Y. Theorical bases of linguistic deficits. Cambridge, MA: MIT Press, 1990.

HAEGMAN, Liliane. Introduction to Government and Binding Theory. Oxford: Basil Black Well, 1991.

LARSON, Rihard K. Bare-NP Adverbs. Linguistic Inquiry, MIT, vol 16 (4): 595621, Fall 1985.

LOBATO, Lúcia M. Pinheiro. Advérbios e preposições, sintagmas adverbiais e sintagmas preposicionais. D.E.L.T.A, vol 5 (1): 101-120, 1989. 
MARANTZ, A. On the nature of gramatical relations. Cambridge, Mass: MIT Press, 1984.

MYSKEN, Pieter e RIEMSDIJK, H. Van. Features and projection. Dordrecht: Foris, 1986.

OUHALLA, Jamal. Functional categories and parametric variation. London and New York: Routledge, 1991.

. Introducing Transformational Grammar- From - Rules to Principles and Parameter. Great Britain: Britishlibrary, 1994.

POLLOCK, Jean-Yves. Extration from NP in French and English: a case study in compartive syntax. 1988

RIEMSDIJK, H. Van. A case study in syntactic markedness: the binding nature of prepositional phrases. Dordrecht: Foris, 1982.

URIAGEREKA, Juan. Warps. 1995. (Mimeograf.).

WILLIAMS, Edwin. The anaforic nature of -Roles. Linguistic Inquiry, MIT, vol 20 (3): 425-56, 1989, Summer 1989.

WILLIAMS, Kemp e DUBINSKY, Stanley. Recategorization of prepositions as complementizers: the case of temporal prepositions in English. Linguistic Inquiry, MIT, Vol 26 (1): 125-137, winter 1995. 